its violence, would exhaust itself before any prudent emetic could operate. It would be easy but tedious to go through writers on the diseases of children, and to show how much at a loss they leave us for a principle on which to treat this "formidable and terrifying disease", as one of them aptly calls it. Miller, Parr, Good, Marsh, Clark, Ley, Evanson and Maunsell, Watson, West, Williams (the latter of whom is the most particular in his directions), insist upon various means - gum lancing, garlic, assafotida, emetics, purgatives, fresh air, opiate and tobacco enemata, but they name no principle to be aimed at in the administration of them; the two latter seem only given as occasional remedies for violence or frequency of paroxysm.

If we have been correct in fixing upon teething as the one exciting cause of laryngismus stridulus; correct also in concluding that it becomes operative only through the excitability which is natural to infancy, but obtains in an exaggerated degree in certain individuals, it results that after the removal of the exciting cause, and taking care that no irritability of the visceral nerves shall add fuel to the fire; in fact, after lancing the gums, effecting a clearance of the bowels, and contriving a prescription, dietetic and meaicinal, for the correction and prevention of acrimony in the bowels, we are left to what I believe to be our most important object, namely, the subjection of nerve excitement by means of narcotics : we are left, in short, to deal with the mairspring of the disease, its predisposing cause. Upon the narcotic to be used, upon the mode of giving it, or the dose required, I need lay but little stress, especially since it is well known how varied is the susceptibility of individuals in reference to this class of medicines. Suffice it to say, that the drug I have most used is opium; that, beginning with small doses, and cautiously regulating them according to their effect, I do not stop short of producing a constant drowsiness and some slight pallor; that when this state is obtained, the paroxysms decrease in force and frequency, the infant is calmly sleeping its day away, no longer devil-torn, nor are its friends racked with anxiety ; and that when the paroxysms have failed to occur for some forty-eight hours, which will usually happen in from three to six days, the drug is gradually withdrawn, the quantity taken off each dose being immediately restored if the attacks show the slightest disposition to encroach again. I may say, also, that to attain to the required effect I have usually been obliged to give to children four or five months old (the age at which the disease most commonly, perhaps, invades) from one to five minims of tincture of opium in a dose with four or six of sal volatile two or three times a day; or if in enemata, five to seven minims.

The principle, then, I would enunciate as the object of treatment after the more obvious ones, the removal of all causes of irritation, is the quieting of nerve excitability - the drowning of hyperæsthesis in a guarded, gentle, yet decided narcotism. I think it probable I may find that there is nothing new in the treatment itself; that, in fact, others have for years pursued the same. It seems to myself so obvious a course that I should be almost surprised to find that it had struck me alone as the proper one. It is, however, the first time, as far as I know, that this principle has appeared in public.

I have depended upon it for the period of twenty years and more; I cannot add, without losing a case; but I can say that it has saved many, or seemed to do so; and with the strictest investigation memory is capable of affording, I see no reason to doubt its safety, its efficiency, or the prudence of continuing it. I therefore adopt it in every instance that presents itself. It is now some years since I named it at the meeting of the Provincial Medical Association held in Wor. cester.

\section{ON SOME OF THE MODES OF ACTION OF IODIDE OF POTASSIUM.}

By E. H. Sieveking, M.D., F.R.C.P., Lecturer on Materia Medica; Assistant-Physician to St. Mary's Hospital, etc.

[Read before the Harveian Society, February 5th, 1857.]

OF the remedies which we owe to the advancenient of modern chemistry, there certainly is none which has obtained a higher place in the estimation of medical men than the iodide of potassium. There may be some difference of opinion as to its efficacy in this or that individual case or in certain classes of disease, but its general utility as a member of the Pharmacopœia is undenied and, I would add, undeniable. Rarely in- ducing any unpleasant effects and, unless administered with culpable carelessness, never giving rise to disturbances in the system of a dangerous and permanently injurious character, it may be classed among the safest and at the same time most beneficial remedies we possess. At times it induces, in ordinary doses, frontal headache and coryza, an eczematoid eruption, or brief salivation; but by diminishing the dose or persevering steadily in the remedy prescribed, we generally soon succeed in removing these symptoms, and the toleration being established, it appears to work for the good of the patient.

Having been called upon unexpectedly and at a very brief notice to furnish a paper for the Harveian Society, it will be impossible for me to enter fully into the various questions connected with the physiological and therapeutic action of this drug. At any time, such a subject could not be exhausted in the course of a single evening. It is my wish to present it to you under some of those aspects, under which I have very frequently witnessed its effects; the more so, as by so doing, I feel satisfied that $I$ shall succeed in eliciting valuable information from the experienced members of the Society present.

The action of iodide of potassium may be defined, according to the more old-fashioned terminology of therapeutics, as al. terative ; and alterative most undoubtedly it is, for it appears to search out the malady which it is intended to attack, and effectually to alter the condition of the solids and the fluids which induces the morbid symptoms. The terminology of therapy now more in vogue would class it among eliminants; and "eliminant" it indeed deserves to be called, for it stimulates powerfully at least one, not the least important secretion of the body, and by promoting the action of the kidneys, causes an elimination of organic and inorganic poisons through their agency. According to another view, the iodide of potassium might be ranked as a tonic, and if to rouse the lymphatic and glandular system from a state of torpor, to promote the removal of sluggish enlargements, to brace the pulpy, flabby tissues of the scrophulous, be a characteristic of a tonic medicine, we may fairly class it under that head. Our object, however, is not to determine what exact place it may occupy in the materia medica, where so much is yet wanting to establish anything approaching to a scientific system; we would rather examine into its action in certain classes of morbid affections, by which we may illustrate the therapeutic effects of the agent. While we admit that this $\dot{a}$ posteriori mode of viewing the subject is not the best possible, we think that in the present state of medical science it is perfectly justifiable. Though it is necessary to remember that the method of arguing from effects, involves those errors to which the manifold interpretation to which morbid conditions are liable, must always tend to expose the deductions of the physician.

Of the various organs of the body, the kidneys most uniformly, as far as we know, demonstrate the action of the salt in question. We trace it by the well known tests in the urine, after the first dose; I have found it in the urine, after a course of iodides, forty hours after the exhibition of the last dose. It rarely fails to increase the quantity of the urine dis. charged, while it manifestly augments the amount of the solid constituents contained in the secretion. The urates, and the uric acid more especially, are evolved in larger quantities, and taking the urine as our test, we clearly recognise a material augmentation in the tissue changes excited in the organism. These tissue changes are rarely otherwise than for the benefit of the patient; they, with rare exceptions, stop short of such destructive processes as are set up by some other minerals; yet it is to be borne in mind that even the iodide of potassium may by too long continued exhibition prove a poison, and by inducing excessive metamorphosis of the tissues, set up a wasting azoturia. Those who have had to deal with such cases know how depressing they are to the patient, how tedious to the physician; but fortunately they are comparatively rare, and with ordinary care these, and even the temporary ill effects of iodide of potassium before alluded to, may be avoided.

I would propose to offer for your consideration the iodide of potassium under three different points of view, as applied to the treatment of disease. It is, however, to be observed that this by no means embraces all its therapeutic aspects.

The modes in which $I$ will regard it, are:

I. As an evacuant of excessive morbid secretions in the cavities of the body.

II. As an antidote to organic poisons.

III. As an antidote to and eliminant of inorganic poisons.

r. Iodide of Potassium an Evacuant of excessive Morbid Secretions in the Cavities of the Body. While I would, in refer- 
ence to the first class of cases, regard iodide of potassium mainly as an evacuant, I would guard myself against the supposition of ever viewing it exclusively in that light. It would appear under all circumstances to act differently from remedies which, like elaterium for instance, eliminate simply by exciting very copious discharges from the system. Even where its evacuant influence is the most palpable, the eliminating action does not always appear to be in the exact proportion of the amount of disease to be removed, but by its stimulant or tonic properties it corrects that morbid condition of the blood and the bloodvessels which led to the morbid product. 'The following case may serve as an illustration to these remarks, while it affords an instance of the class of cases in which the eliminant influence of the iodide is most strongly marked. It is a case of hydrothorax, of a subacute character.

CASE. J. M., the son of a carpenter, aged 7 years, was brought to me, with pain in the right hypochondria, attributed to a fall from a cart, which had happened eight or nine days previously. There was some cough. The right side of the thorax was enlarged, though the intercostal spaces did not project. Scarcely any movement was perceptible during respiration, in this side; it was absolutely dull throughout, anteriorly and posteriorly, except at the suprascapular space; this was the only point at which a vocal fremitus could be perceived, and that disappeared on lying down. Tubular breathing was found at the apex of the right lung, and diminished gradually downwards. The child suffered from dypsnœa, and had a distressed and anxious appearance. His pulse was 84 . On the 11 th Sept. 1855 , I prescribed as follows :

R Potassii iodidi 3 ss; spiritûs etheris nitrici 3 ii; mist. camph. żviii. M. Fiat mistura cujus sumat 3 ii tertiis horis.

Applicetur emplastrum vesicatorium magnitudinis $3 \times 3$, lateri thoracis dextro.

Sept. 14th. He was very much better, the appetite and breathing better, though the blister had not drawn. The bowels were open; discharge of urine free.

Sept. 18th. The patient was so much improved as to walk to see me; his cough was rather more frequent, but there was no expectoration; he was passing much urine; the bowels were open; the general appearance better and fuller; the dullness was diminishing from the apex downwards, and the dullness of the anterior thorax generally was diminished in the recumbent position. 'The tongue was furred at the root. The patient had slept better for three nights past. He continued taking the same medicine, improving in appearance and gaining flesh.

Oct. 2nd. The thorax is described as resonant, and present. ing a fair respiratory murmur as far as the nipple on the right side. On deep inspiration, the respiratory murmur could be traced further still; below the nipple, it was tubular. A friction sound was heard about the middle of the outer side of the thorax. It was noted that the lungs were descending, and the fluid being absorbed.

Oct. 30th. There was still some dullness found over the lower third of the right lung, the breathing was normal below the scapula; anteriorly under the right nipple it was slightly bronchial, and the heart sounds were increased at the same point. The boy looks robust, has a good complexion and excellent appetite; the tongue is whitish; the pulse, 108. The medicine was now changed to the syrup of iodide of iron half a drachm in water three times a day, which he took until the 4th December. He then received cod liver oil, which he took to the 18th December, when the lad was discharged cured.

It will be observed that no other remedy was administered throughout the period during which the effusion into the pleural cavity formed the corpus delicti, and it may therefore be fuirly assumed that the removal of the fluid was due to the iodide of potassium ; hydrothorax not being one of the diseases which have a tendency to spontaneous cure. But I could bring forward other proofs of a similar stringency, though in the majority of cases we are not satisfied with trusting to one remedy only, but consider a multiplication of our agents necessary to reduce the fortress of the enemy. To continue the simile, we may urge that in our warfare, we too often do not rest satisfied with destroying or rendering innocuous the hostile battalions, but must needs batter down the strongholds as well, and throw our hot shells into his magazine so as to cause a grand explosion, and a blaze of light, followed by a no less deplorable darkness and desolation. In effusions into serous membranes dependent upon organic disease of an important viscus, the efficacy of iodide of potassium varies, and as a rule we should be less disposed to rely upon it, than upon other remedies. I have no experience of it in renal disease, where it would seem to be generally contraindicated, the more so as we rarely have to deal with morbid local conditions of the kidneys, until the disorganisation has proceeded too far to be benefited by this agent. Moreover, the undoubtedly stimulant influence it exerts upon the kidneys would contraindicate its employment wherever an increased afflux of blood to the organs would be prejudicial.

We would say one word upon the influence of iodide of potassium upon cerebral hrmorrhage. Its action in these cases appears to be analogous to the action manifested in the instance of hydrothorax above detailed, though it may be here sug. gested that the natural tendency of the disease is to spontaneous cure after the first shock has passed off. Still we have seen so many cases, in which a more rapid absorption of the effused blood might fairly be inferred after the administration of iodide of potassium, than seemed to be taking place before it was given, that we cannot but regard it as one of the most valuable aids we possess in promoting the absorption of the extravasated blood, and the consequent removal of the pressure which induced the symptoms of hemiplegia. We have in these cases to deal with an effusion of a different character, and of a much smaller extent than we meet with in serous accumulations; but equally as in the latter, we wish in no way to inter. fere with, but rather to promote, the plasticity of the blood; hence we cannot but regard a systematic exhibition of mercurials in these cases as most reprehensible; while the iodide of potassium, if it may not prevent the renewed effusion into the cerebrum, will not at all events impoverish the blood and thus facilitate a return of the hæmorrhage.

Ir. Iodide of Potassium as an Antidote to Organic Poisons. We here trench more upon hypothesis than either in the point of view under which we have just examined the salt, or under that to which I shall direct your attention in the third instance. Yet in some cases the presence of an agent that contaminates the entire fountain of life is so palpably manifest, that we appear justified, by analogical reasoning, to infer the presence of a similar agent in other cases, which do not admit of being traced back with equal certainty to an organic poison. The syphilitic poison is undeniable, whether it manifest itself in the primary chancre, the contagious bubo, the ulcerated sore throat, the cupreous eruption, or the flat condyloma; in one sense we can scarcely refuse to admit that the proximate cause of rheumatism is a poison; in many skin diseases of an obstinate and a recurrent character, the evidence at our disposal tends to prove that a poison circulates in the system, which may periodically fall into a state of abeyance, but which, unless searched out, and neutralised by a more powerful agent, breaks out every now and then, to the annoyance of the patient and the disappointment of the medical attendant. Lepra and psoriasis appear to bear this type in many cases. The skin diseases that present the same outward characters often differ materially in their proximate cause and in their relation to the constitution of the individual; hence it is as erroneous to apply a uniform remedy to all scaly, all herpetic, or all impetiginous eruptions, as it would be to treat all cases in which we hear a crepitant noise in the thorax with the same tartrate of antimony mixture or Plummer's pills. It is impossible to give ocular demonstration of the organic poisons, but of the existence of some peculiar compound which is amenable to treatment by iodide of potassium in many of the cases alluded to, we entertain no doubt. In the instance of rheumatism, the existence of an organic acid in the blood appears to constitute the materies morbi which may either be decomposed or be ejected from the system, but in both cases rendered innocuous under certain circumstances by iodide of potassium. Where much depends upon analogical reasoning, the diagnosis of a chronic affection is necessarily rendered a matter of difficulty, and it is a severe test of medical ability. In many cases, we may be compelled to reserve our diagnosis until we have brought the guns of the Pharmacopoia to bear; there is this advantage in employing iodide of potassium as a test, that it will not be used at any serious risk to the patient, but will very probably prove beneficial

There is some difficulty in selecting cases in illustration of the view we are now taking, since it rarely happens that in the very chronic cases to which we allude, it is wise to adhere to the use of one remedy throughout. Consequently, a person disposed to refuse credit to the remedy in question, may attribute the curative influence to another agent. The conviction which a multitude of individual experiences and experiments forces upon the practitioner, cannot easily be conveyed by the quotation of a single case.

IIr. Iodide of Potassium as an Antidote to and Eliminant of Inorganic Poisons. The cases of metallic poisoning which most 
frequently come before us, are those of artificers who are employed with lead in some form or other, most commonly as the acetate or white-lead, and thus receive the poison in small quantities, gradually absorbed, into the system. Painters necessarily are most liable to suffer in this way; the facility and rapility with which the system becomes affected depends upon a variety of circumstances; the vigour of the patient's constitution, his habits of cleanliness, the purity of the atmosphere in which he habitually works. The most rapid ease of saturnine intoxication that has occurred in our own experience, was in á man not generally employed in this way, who for three dars was engared in applying a new process to the fastening of a roof, in which red lead was employed; during the whole time he was in a confined locality and forced to respire the fumes of the lead, and at the end of the three days gave unequivocal signs of lead-poisoning. The blue line which was shown by Dr. Burton to fringe the gums of persons poisoned by lead, and possibly dependent upon the formation in the system of a sulphuret, is an almost invariable characteristic of the affection, yet cases do occur where after exposure to the poisonous influence, symptoms are manifested which resemble lead-poisoning, and yield to the iodide, though the blue line is absent. Of the rapid astion of iodide of potassium in saturnine neuralgia, the foliowing are instances.

Cass. George C., aged $2 \pi$, a painter, had colic two months previous to coming under my care. He then had been affected with it for six days, and was slightly jaundiced. His belly was hard, but towards night it became as "hard as a stone." There was no tenderness. A slight blue line was seen to edge the gums.

Sept. 15th. The following medicine was ordered:

Be Potass. iodidi gr. v; liquoris opii $m \mathrm{x}$; misture camph. $\zeta \mathrm{i}$.

The pain ceased two days after commencing the medicine. The liquor opii was then omitted. On Sept. 26th, it is stated that the colic is much better; that there is still occasional pain in the upper left abdomen; that the blue line continues. I then gave him ten grains of iodide of potassium, and three weeks later there had been no return of pain.

The following is asimilar instance.

CASF. Thomas L., aged 28, painter, had never had colic or dropped hands, till a fortnight before coming under treatment he was attacked with severe and constant pain about the navel; the parts were tender; sense of nausea; urine highcoloured; bowels costive. A trace of blue line round several teeth. P'ulse 72 , normal. Good appetite.

Dec. 12th. The following pills were ordered to be taken every night at bed time.

B Pilula colocynthidis comp. gr. $\mathrm{x}$; olei crotonis gtt. i.

Three days later, the pain is stated to be less; the bowels had been moved twice each day. The iodide of potassium was now administered in five-grain doses, and three days after the pain had left him, and though under treatment for other affections, for alove two montlis, the pain did not return. The iodide was continued for some time, and when discharged cured, the gums were restored to their normal condition.

Many interesting questions are connected with the action of iodide of potassium in cases of metallic poisoning; not the least important being the mode in which the metal is eliminated. That it passes off by the urine has been demonstrated; but it is manifestly eliminated by other channels, because, at times, during its administration in undoubted cases of leadpoisoning, while the patient is rapidly recovering, the lead cannot be detected in the urine. We must then assume that it passes off by tho bowels, after being set free by the antidote. Nor is the detection of the lead itself in the urine so easy a process; it does not occur in the form of an ordinary salt, but appears to enter into a very intimate combination with the organic matter of the urine, which must be destroyed before the common tests scrve to demonstrate it.

In an rtiological point of view, the study of lead-poisoning is extremely interesting, as it gives rise to the most varied forms of nervous disease, in all of which an accurate diagnosis is the more important, as the proper remedy will rarely fail to establish a cure, that remedy being iodide of potassium. Thus epilepsy, almost alwars a disease with a doubtful prognosis, appears to lose this character when we trace it to lead-poisoning; for the cause may be removed, and with it the demoniac seizures likewise disappear. Steel and quinine, our ordinary anchers in neuralgie affections, fail us in lead-poisoning; but we are sure of allaying the pain as soon as we know that it is a case for iodide of potassium.

There is another metal which, to the disgrace of our profes- sion, has long been employed to poison our fellow-men, under the protection of the rgis of science, but which now appears to be placed under such restrictions as to render it truly a remedial agent: we allude to mercury. This drug also, in its poisonous effects, finds a powerful and certain antidote in iodide of potassium. The latter searches it out in its various combinations with the organic constituents of the body, and, after more or less resistance, effectually expels it. Some pain most generally results from the mercury being first set free; and even salivation, which had subsided, may thus be again excited ; but, in salivation itself, we know of no more effectual remedy, besides the exhibition of local astringents (upon which, however, we would not lay much stress), than the iodide.

We will close this paper by quoting a case in illustration of its efficacy under these circumstances.

James C., a painter, who had generally enjoyed good health, had been taking flowers of sulphur during the preceding summer, to counteract the effects of the lead paint with which he worked, but had never had the colic or dropped hands. Two weeks previously, his face began to swell. At first there was sore throat; then the gums swelled; and the teeth, which previously had been sound, became quite pulpy. The gums, a the time of consultation, were invisible, from the tumefaction of the adjoining parts. There was a decided mercurial fotor and the man bore the stamp of a man who was salivated; still he denied having taken any mercurial, and ascribed his affection to using a vinegar and rye gargle, and to working in a damp place. However, I prescribed-

R Potassii iodidi gr. iv; infusi cascarillæ 3 j. M. Fiat haustus ter die sumendus.

Sumat olei ricini そss omni mane.

Habeat decocti quercûs $\mathrm{Oj}$ pro gargarismate.

At the next visit, he, on repeated questioning, admitted having been in the habit of occasionally taking some pills prescribed for him by a physician a long time ago, and which contained some hydrargyrum cum cretâ. The treatment was steadily persevered in, and the improvement was satisfactory. In the third week, the report is, "Doing well; teeth recovering their normal appearance, but still loose, though less than they were; there was a blue ring round the gums." He now returned to work; the mercurial foetor soon entirely disappeared; and in another month he was able to masticate.

In conclusion, allow me once more to revert to a remark made at the earlier part of my paper, that my intention has not been to exhaust the subject, as I know full well that much more might be said upon the action of iodide of potassium in other maladies than those named. My desire has been to submit for your consideration some of the opinions which practical experience has induced me to form relative to the drug we have considered, and to elicit from the stores of your own observation further illustrations in regard to its use and effects.

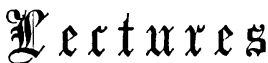

\section{ON CERTAIN VIEWS ON THE NATURE AND} TREATMENT OF PHTHISIS PULMONALIS.

DELIVERED AT THE

BROMPTON HOSPITAL FOR CONSUMPTION AND DISEASES OF THE CHEST, IN THE SUMMER OF 1856.

By EDWARD SMITH, M.D., LL.B., B.A., Licentiate of the Royal College of Physicians; Assistant-Physician to the Hospital, etc.

LECTURE III (concluded).

REMOVAL OF THE EFFECTS OF THE DISEASE BY ABSORPTION AND EXPECTORATION. GENERAL SYMPTOMS : PULSATION, HEMOPTYSIS, COUGH, DYSPNCEA, NIGHT-SWEATS, WASTING, AND DEBILITY. MODE OF ACTION OF COD-LIVER OIL.

DEBILITY AND WASTING are invariable concomitants of the disease in some of its stages; and it is highly probable that the former may in many instances be both a cause and an effect. Neither, however, necessarily precede phthisis, nor indeed necessarily attend the early stage of the disease. As a cause, debility acts, as has already been pointed out, by lessening the intensity of respiration; or, in other words, both the debility and the lessened respiration are due to or connected with lessened nervous influence. Thus it is that systems of defective organisation, as those of the scrofulous diathesis (if we would refer to a large and heterogeneous class in illustration), are so 